\title{
Editorial
}

\section{Special issue on learning and control in cooperative multi-agent systems}

Cooperative control of multi-agent systems linked by communication networks is a well-developed and still growing field. The interplay of the individual agent dynamics and the communication graph topology results in intriguing and often surprising behaviors that are not manifested in the study of control systems for single-agent dynamics. This field brings systems theory, feedback control, graph theory, communication systems, complex systems theory to provide rigorous analysis and design for multiple dynamical systems interconnected by a graph information flow structure. Applications have been made to vehicle formation control, coordinated multi-satellite control, electric power system control, robotics, autonomous airborne systems, manufacturing production lines, and the synchronization of dynamical processes in chemistry, physics, biology, and chaotic systems.

The design of systems that learn online how best to control dynamical processes is well developed. Adaptive control is a proven method for learning feedback controllers for systems with unknown dynamic models, exogenous disturbances, nonzero setpoints, and uncertain nonlinearities and unmodeled dynamics. Adaptive control has been applied for years in process control, industry, aerospace systems, vehicle systems, and elsewhere. Reinforcement learning (RL) refers to a broad class of methods for improving control policies based on observation of the performance or value of current policies. RL allows the learning of optimal controls in real-time using data measured along system trajectories. RL is closely tied theoretically to both adaptive control and optimal control. Recent work has shown how to use RL to develop new forms of adaptive controllers that converge to optimal control solutions in real time and to effectively deal with some existing open problems in adaptive control such as handling unmatched uncertainties. Developed methods include approximate dynamic programming, integral reinforcement learning, temporal difference, adaptive function approximation, and others.

However, the design of cooperative control systems with real-time learning features has not been fully developed. The purpose of this special issue is to present a body of work that shows how to more closely integrate and cross-fertilize techniques from cooperative control, adaptive control and RL. Indeed, the special issue consists of four papers among which one discusses the pinning synchronization of networked multi-agent systems, one studies dynamic graphical games using reinforcement learning methods, one considers the formation control problem of a group of agents, and one deals with flocking problem of nonlinear agents using optimistic planning. All papers were selected from numerous submissions and carefully reviewed. We hope that the special issue will promote the research interest in learning and control in cooperative multi-agent systems.

Guest editors:

Frank L. Lewis

The University of Texas at Arlington, Texas, U.S.A.

Zhong-Ping Jiang

New York University, NY, U.S.A.

Tengfei Liu

Northeastern University, Shenyang, China

(c) 2015 South China University of Technology, Academy of Mathematics and Systems Science, CAS, and Springer-Verlag Berlin Heidelberg 